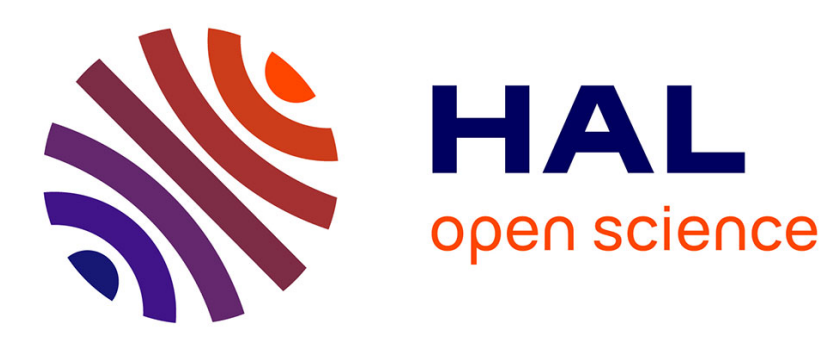

\title{
Implicit integral equations for modeling systems with a transport delay
}

Delphine Bresch-Pietri, Nicolas Petit

\section{To cite this version:}

Delphine Bresch-Pietri, Nicolas Petit. Implicit integral equations for modeling systems with a transport delay. E. Witrant; E. Fridman; O. Sename; L. Dugard. Recent results on time-delay systems: analysis and control, 5, , 2016, Analysis and Control, 978-3-319-26367-0. 10.1007/978-3-319-263694_1. hal-01384486

\author{
HAL Id: hal-01384486 \\ https://hal.science/hal-01384486
}

Submitted on 20 Oct 2016

HAL is a multi-disciplinary open access archive for the deposit and dissemination of scientific research documents, whether they are published or not. The documents may come from teaching and research institutions in France or abroad, or from public or private research centers.
L'archive ouverte pluridisciplinaire HAL, est destinée au dépôt et à la diffusion de documents scientifiques de niveau recherche, publiés ou non, émanant des établissements d'enseignement et de recherche français ou étrangers, des laboratoires publics ou privés. 


\title{
Implicit integral equations for modeling systems with a transport delay
}

Delphine Bresch-Pietri and Nicolas Petit

\begin{abstract}
In this chapter, we present a particular class of transport delay systems (e.g. systems involving transportation of material), in which the delay is defined through an implicit integral equation. To illustrate the practical interest of this class, experimental use of such models is presented for two different examples of physical systems, both from the field of automotive gasoline engines (specifically, exhaust gas recirculation and exhaust catalyst thermal dynamics). We also discuss related control challenges, together with some solutions.
\end{abstract}

\section{Some motivations for investigating transport delay modeling}

Time-delay systems have been widely investigated in the past decade following the rise of telecommunications and network exchanges. Due to the practical relevance of such cases, this research effort has yielded a substantial number of monographs and studies devoted to time-varying delays $[4,31,44]$. In this field of research, the variability of the studied delay is usually unstructured. Another important class of delays consists in the ones arising from transportation of material. Prime examples of such physics-driven systems are mixing processes [35] for liquid or gaseous fluids, chemical reactors [17], automotive engine and exhaust line [21], heat collector plant [38], and blending in liquid or solid networks [10], to name a few.

Despite this record, control oriented modeling and control design for transport delay systems is still an underdeveloped field. The varying delays are either represented by purely uncertain time-varying models or, in the worst case, by a constant

Delphine Bresch-Pietri

GIPSA-lab, Department of Automatic Control, 11 rue des Mathématiques, 38000 Grenoble, France e-mail: delphine.bresch-pietri@gipsa-lab.fr

Nicolas Petit

MINES ParisTech, PSL Research University, CAS - Centre Automatique et Systèmes, 60 bd StMichel, 75006 Paris, France e-mail: nicolas.petit@mines-paristech.fr 
mean value. Yet, in all the above mentioned applications, the lag variability is strong, especially because it depends on the past values of the control variable ${ }^{1}$.

This chapter aims at filling the gap in this area by stressing the relevance of a particular class of time- and input-dependent delay defined through the following implicit integral equation

$$
\int_{t-D(t)}^{t} \varphi(s, u(s)) d s=1, \quad \varphi>0
$$

In this equation, which covers the examples mentioned above, the delay $D$ appears in the lower bound of the integral and $\varphi$ is a strictly positive function that depends on the manipulated variable $u$ and on the time. Such a model is commonly used in process and chemical engineering industry (see [45] or recently [42]) and even owns a dedicated block in the simulation software Simulink ${ }^{\circledR}[30]$, Variable Transport Delay. Nevertheless, it is infrequently employed in the control community. Further, the input-dependency of this model is often neglected and has seldom if never been studied theoretically (see the corresponding discussion in [37], in which the crushing-mill example that is outlined fits into the framework of this chapter).

In this chapter, the model (1), proposed for the first time in [46] for plug flow vessels up to the authors' knowledge, is highlighted and shown to be representative of a wide class of systems involving transport of material. To illustrate its practical interest, experimental use of this model is presented for two systems from the field of automotive gasoline engine: the temperature at the exhaust of the catalyst, and the exhaust gas recirculation. Even if control design is not directly in its scope, related challenges are also discussed, together with some potential solutions.

The chapter is organized as follows. In Section 2, the transport delay model (1) is presented in details and discussed. Then, after some elements of context, Section 3 focuses on the design of a control-oriented model for catalyst temperature, in which a transport delay appears. Section 4 proposes to apply this model to capture the dilution dynamics occurring in a gasoline engine equipped with burned gas external recirculation loop. Finally, as a conclusion, control stakes are exposed in Section 5.

\section{Implicit integral delay equations}

The implicit integral equation (1) defines a transport delay, in which $\varphi$ should be understood as a normalized velocity. To illustrate this point, we formulate the following result.

Lemma 1. Consider a fluid flow with varying speed $v(t)>0$ through a pipe of length L, as depicted in Figure 1. The propagation time D of the fluid through the pipe, if finite, is defined according to the integral equation

\footnotetext{
${ }^{1}$ In particular, if the input varies to counteract the lag effects by means of anticipation, the lag varies in an implicit and possibly malicious fashion. This raises concerns about stability, in both open and closed loop scenarii.
} 


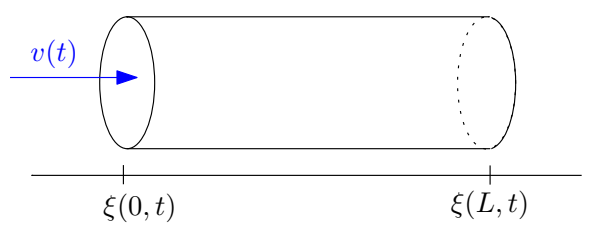

Fig. 1 The transport of a variable $\xi$ with a varying speed $v(t)$

$$
\int_{t-D(t)}^{t} v(s) d s=L
$$

Proof. Formally, this integral equation can be directly obtained by observing that the quantity $\xi(x, t)$ travelling through the pipe satisfies the transport PDE

$$
\xi_{t}+v(t) \xi_{x}=0, \quad x \in[0, L]
$$

with $v(t)>0$. Consider a given time $t \geq 0$ and, for $\tau \geq t$, the change of variable

$$
w(\tau)=\xi\left(\int_{t}^{\tau} v(s) d s, \tau\right)
$$

which satisfies $w_{\tau}=0$. Therefore, $w(\tau)=w(t), \tau \geq t$. Now, if it exists, consider $r(t)$ such that

$$
\int_{t}^{r(t)} v(s) d s=L
$$

and, otherwise, define $D(t)=\infty$. Taking a derivative of this relation with respect to $t$, one can obtain that $r$ is invertible as $\frac{d r}{d t}=\frac{v(t)}{v(r(t))}>0$. Therefore, introducing $D(t)=t-r^{-1}(t)$, this relation can finally be reformulated as

$$
\int_{t-D(t)}^{t} v(s) d s=L
$$

which is the integral relation (2). The proof is complete by observing that, either $D(t)=\infty$, or

$$
\xi(L, r(t))=w(r(t))=w(t)=\xi(0, t)
$$

which is equivalent to $\xi(L, t)=\xi\left(0, r^{-1}(t)\right)=\xi(0, t-D(t))$, i.e. the delay $D(t)$ between the output and the input of the system satisfies (2).

Therefore, the delay induced by the transport of a fluid flow with varying speed $v(t)>0$ through a pipe of length $L$ belongs to the class of model (1), with $\varphi(s, u(s))=\frac{v(s)}{L}$. When the flow speed depends on the input, this delay in input- 
dependent. The model (2) can be interpreted as the generalization of an intuitive propagation time model, for which the delay is defined as the ratio between the length of the pipe and the gas speed. This situation corresponds to the steady-state case, for which the gas speed is constant and is indeed caught by (2).

\subsection{Properties}

Property 1. The delay defined through (1) is indeed a transport delay in the sense that $D(t)>0$ and $\dot{D}(t)<1$ for $t \geq 0$.

The second property guarantees that the plant is causal, i.e., that no input values older than the ones that have already reached the system can reach the plant, as $\frac{d}{d t}(t-D(t)) \geq 0, t \geq 0$. In other words, the input signal direction is never reversed, which ensures that the physical phenomenon is causal.

Proof. The positivity of $D$ follows from the one of $\varphi$. Indeed, if $t-D(t) \geq t$, then the left-hand side of the equation is non-positive, which is absurd. Second, taking a time-derivative of (1), one can obtain

$$
1-\dot{D}(t)=\frac{\varphi(t, u(t))}{\varphi(t-D(t), u(t-D(t)))}>0
$$

as $\varphi$ is a strictly positive function.

\subsection{Numerical calculation}

As this point is crucial in practical applications, it is worth highlighting the fact that, even if the transport delay defined through (1) cannot be analytically expressed, as underlined in [46], it can still be calculated numerically if the history of function $\varphi$ is known ${ }^{2}$.

Indeed, the function $D \mapsto \int_{t-D(t)}^{t} \varphi(s) d s$ is a strictly increasing function, equal to zero for a zero delay. Therefore, a simple procedure to evaluate the current value of the delay consists in evaluating the value of (a sampled version ${ }^{3}$ of) this function for increasing delay values, starting with $D=0$, until reaching a certain bound ${ }^{4}$, say the value 1 . Such a procedure, which is real-time compliant, is illustrated in Table 1.

${ }^{2}$ The procedure proposed here is not exactly the one proposed in [46], which relies on z-invariance. ${ }^{3}$ The sampling method may not involve $u(t)$ if the control law is computed according to the delay value.

${ }^{4}$ If reachable, which is the case for example if $\varphi$ has a strictly positive lower-bound. Otherwise, $D$ can be evaluated as infinite after a certain number of iterations. 


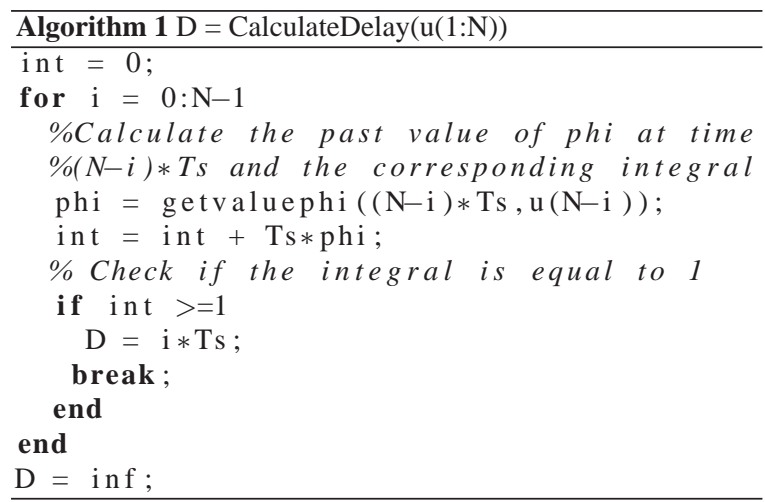

Table 1 Example of delay calculation procedure for the integral-type relation (1). Purposely, the integral sampling does not involve the current input value $u(t)$.

We now present two examples of physical systems that involve transportation of material and for which the resulting delay transport can be modeled according to (1).

\section{Application to the design of a control-oriented temperature model for Spark-Ignited engine exhaust catalyst}

\subsection{Context and motivations}

Automotive Spark-Ignited (SI) engines are equipped with a Three-Way Catalyst (TWC) located in the exhaust line. This after-treatment device aims at reducing pollutants resulting from the combustion. Yet, conversion efficiency highly depends on the catalyst (distributed) substrate temperature [19,22]. Therefore, it is of critical importance to control the combustion according to the catalyst temperature state, in particular during light-off phases after a cold start. Nevertheless, no sensor is commercially embedded to provide such an information and it is necessary to rely on a model to perform this task.

Unfortunately, existing catalyst temperature models proposed in the literature are: either mean-value models [25], which do not take into account the inherent distributed nature of the catalyst and can therefore reveal highly inaccurate (when one is interested in the temperature at a given location, $0 \mathrm{D}$ models can be tuned accordingly and reveal satisfactory for control purposes. Yet, to account, e.g., for the catalyst ageing, it may be useful to estimate its temperature at different locations, which cannot be done with sufficient accuracy with such a lumped model); or Partial Differential Equations (PDE) [12,26,33], discarded from real-time implementations by their induced computational burden. 
Here, in view of obtaining a model of limited complexity, it is taken advantage of the fact that, as this system involves transportation of material (exhaust gas), it can then be included in the vast class of flow processes covered by this chapter and represented by a transport delay of type (1). This result is obtained by designing a semi-lumped approximation of the underlying PDE equations. For sake of conciseness, these design elements are not presented here but the interested reader is referred to previous works detailing them $[8,9]$.

\subsection{Approaching the temperature dynamics by a first-order equation driven by an integral transport delay}

A schematic representation of the thermal exchange occurring inside the monolith is given in Figure 2. Exhaust burned gas enter the monolith at $x=0$ and convective exchange with the wall occur all along the monolith, i.e. for $x=0$ to $x=L$. This yield inhomogeneous distributed temperature profiles for the gas $T_{g}(x, t)$ and the catalyst wall $T_{w}(x, t)^{5}$ which are governed by the following equations [27]

$$
\left\{\begin{array}{l}
\frac{\partial T_{w}}{\partial t}(x, t)=k_{1}\left(T_{g}(x, t)-T_{w}(x, t)\right)+\Psi\left(x, t, T_{w}(x, t)\right) \\
\dot{m}_{g} \frac{\partial T_{g}}{\partial x}(x, t)=k_{2}\left(T_{w}(x, t)-T_{g}(x, t)\right)
\end{array}\right.
$$

where $\dot{m}_{g}$ is the inlet gas mass flow rate, $k_{1}, k_{2}>0$ are given positive physical constants and $\psi$ is a distributed time-varying source term, related to the chemical reaction occurring inside the catalyst.

To represent the source term $\psi$, following $[27,28]$, we propose to consider the pollutant conversion effects as a second temperature front $T_{e q}$ occurring at a virtual position $L_{r}$ inside the catalyst. Indeed, for steady-state conditions, energy balance

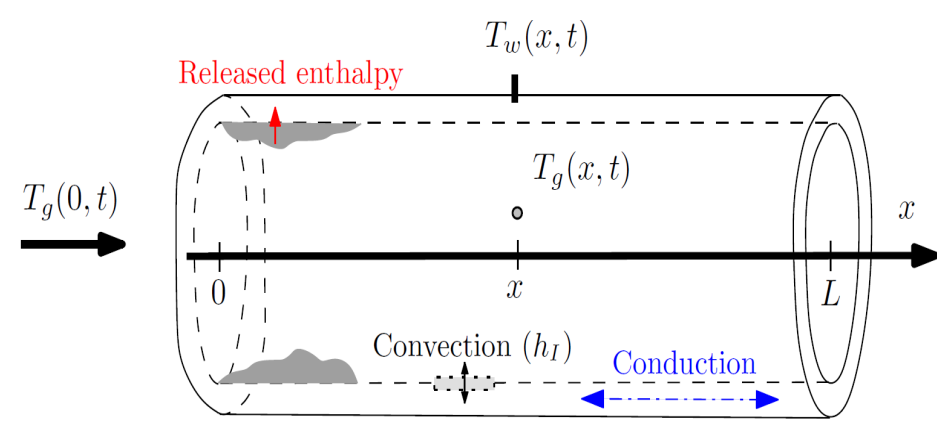

Fig. 2 Schematic view of the distributed profile temperature inside a catalyst jointly with thermal exchanges.

5 The axial conduction in the solid can be neglected $[40,43]$ 
for the system can be written as

$$
\dot{m}_{g} C p_{g} \underbrace{\left(T_{g}(0)-T_{g}(L)\right)}_{\triangleq_{T_{e q}}}+\eta\left(T_{w}\left(L_{\eta}\right)\right) \sum_{i=1}^{N} \Delta H_{i}\left[x_{i}\right]_{i n}=0
$$

where $\left[x_{i}\right]_{i n}$ are the inlet pollutant concentrations, $C p_{g}$ is the gas heat capacity and $\Delta H_{i}$ is the unity enthalpy relative to the conversion of the pollutant $i$. Typically, three main pollutants are considered $(N=3)$, i.e. hydrocarbons $(\mathrm{HC})$, carbon monoxide $(\mathrm{CO})$ and nitrogen oxides $\left(\mathrm{NO}_{x}\right)$. This results in the definition of an equivalent temperature

$$
T_{e q}=\sum_{i=1}^{N} G_{i}\left[x_{i}\right]_{i n}
$$

in which the steady-state gains can be calculated as $G_{i}=\eta\left(T_{w}\left(L_{\eta}\right)\right) \frac{\Delta H_{i}}{\dot{m}_{g} C p_{g}}$. In practice, the pollutant concentrations are not measured but can be effectively estimated, e.g. by look-up tables.

Claim 1 The wall catalyst temperature at position $L_{\eta}$ can be efficiently represented as

$$
T_{w}\left(t, L_{\eta}\right)=T_{w}^{t h}(t)+T_{w}^{\psi}(t)
$$

where $T_{w}^{t h}$ satisfies

$$
\tau\left(L_{\eta}, t\right) \frac{d T_{w}^{t h}}{d t}=-T_{w}^{t h}(t)+T_{g}\left(0, t-D\left(L_{\eta}, t\right)\right)
$$

and $T_{w}^{\psi}$ satisfies

$$
\tau\left(L_{\eta}-L_{r}, t\right) \frac{d T_{w}^{\psi}}{d t}=-T_{w}^{\psi}(t)+T_{e q}\left(0, t-D\left(L_{\eta}-L_{r}, t\right)\right)
$$

in which $T_{e q}$ is given in (5), the time constant $\tau$ and the delay $D$ are defined for $x \in[0, L]$ as

$$
\left\{\begin{array}{l}
\tau(x, t)=\frac{1}{k_{1}}+\nu \delta(x, t) \\
D(x, t)=(1-\nu) \delta(x, t)
\end{array}\right.
$$

with $\nu$ a given constant in $[0,1]$ and $\delta$ defined through the integral equation 


$$
\int_{t-\delta(x, t)}^{t} \frac{k_{1}}{k_{2}} \dot{m}_{g}(s) d s=x
$$

The proof of this claim can be found in [8].

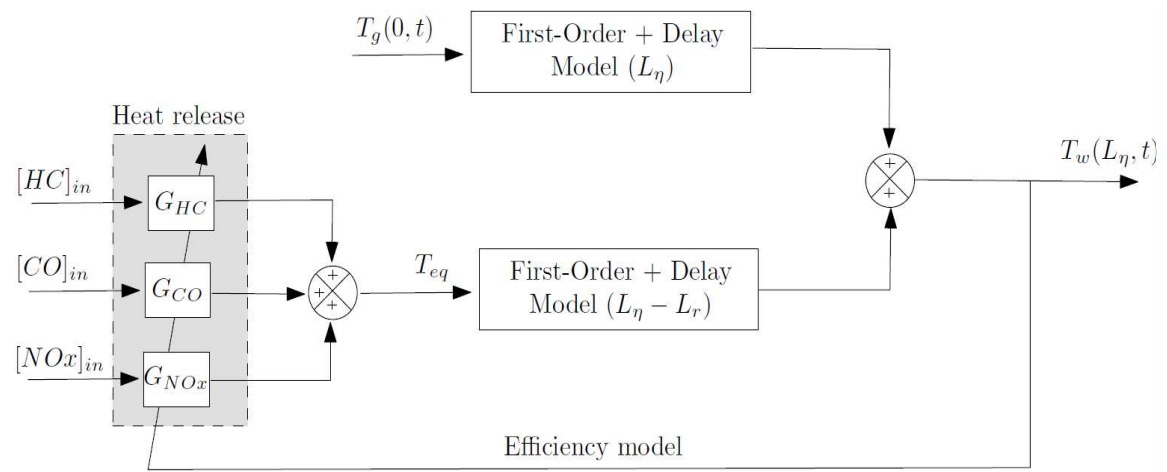

Fig. 3 Proposed catalyst temperature model (6)-(11). The pollutant conversion effect (HC, CO and $\mathrm{NO}_{x}$ ) are assimilated to a front of temperature $T_{e q}$ propagating on a virtual length $L_{\eta}-L_{r}$, while the gas heating occurs on the complete length $L_{\eta}$. The model is also fed by the gas mass flow rate $\dot{m}_{g}$, which is not represented here for the sake of clarity.

The proposed model is grounded on the linearity of the dynamic through a superposition principle: the effects of the inlet temperature $T_{g}(0)$ is distinguished from the one of the pollutant conversion. The distributed temperature of the catalyst is then modeled as the sum of two similar input-delay equations. The propagation phenomena occurs on two different lengths. This model is pictured in Fig. 3.

Relation (11) is in the form (1) with $\varphi(s, u(s))=\frac{k_{1}}{k_{2}} \frac{\dot{m}_{g}}{x}$ which is a positivevalued function. For an hybrid vehicle, the mass flow rate is an actuated variable and this integrand function is input-dependent. Here, this propagation time can be understood as a residence time into the monolith (see [11]). As the two main effects of the gas residence inside the monolith are transport and exchange with the monolith, it can reasonably be separated into a first order dynamics with a pure delay effect.

The tuning parameter $\nu$ and the reactive length $L_{r}$ can be determined via dedicated testings and allows this model to qualitatively represent a relatively vast range of catalyst devices. 


\subsection{Validation of the reduced model (6)-(11) using experimental data}

To illustrate Claim 1, simulation results of the temperature inside the wall catalyst at two different locations are pictured in Figure 4 and compared to experimental data. The inputs used to evaluate the model (gas mass flow rate and gas inlet temperature) are data recorded during the European normalization cycle (NEDC). They are pictured in Figure 4(b). In particular, one can observe that the considered gas mass flow rate variations are quite large, which makes this test challenging, as the model time constants and delays depend on this quantity according to (11).

The simulated temperature in Figure 4(a) almost perfectly matches the experimental data. It is worth emphasizing that such a match could not be obtained with a 0D model which inherently approximates the distributed temperature by an average one: a compromise between representativity of the two temperatures would have to be reached. Further, as these performances are obtained for very demanding external conditions, one can reasonably expect similar behavior on different kinds of driving conditions. Use of this model for light-off strategies are discussed in [9].

\section{Practical implementation for Exhaust Gas Recirculation for Spark-Ignited engines}

We now present a second application from the field of automotive engine, the Exhaust Gas Recirculation for Gasoline engine, for which the proposed delay model is of interest, as will appear in the sequel.

\subsection{Context and motivations}

To prevent the malicious knock phenomenon appearing at high load in downsized Spark-Ignited engines, one of the solutions considered in the automotive industry consists in using exhaust gas recirculation (EGR) through a low-pressure circuit (see [20] or [36]). A typical implementation is represented in Fig. 5.

Yet, EGR has strong interactions with other simultaneously operating engine controllers such as the regulation of Fuel-to-Air Ratio (FAR) to stoichiometry (see [21]). To counteract the impact of intake burned gas, a solution would be to modify the feedforward action on the cascaded controllers (fuelpath controller and ignition path controller) based on a real-time estimate $\hat{x}$ of the intake burned gas rate. Nevertheless, no real-time sensor of this variable is embedded in any real-world vehicle.

For this reason, the approach employed here is to substitute one such sensor with a model. Again, this systems fits into the general framework of flow processes, as it involves transportation of burned gases on a relative long distance (between the 

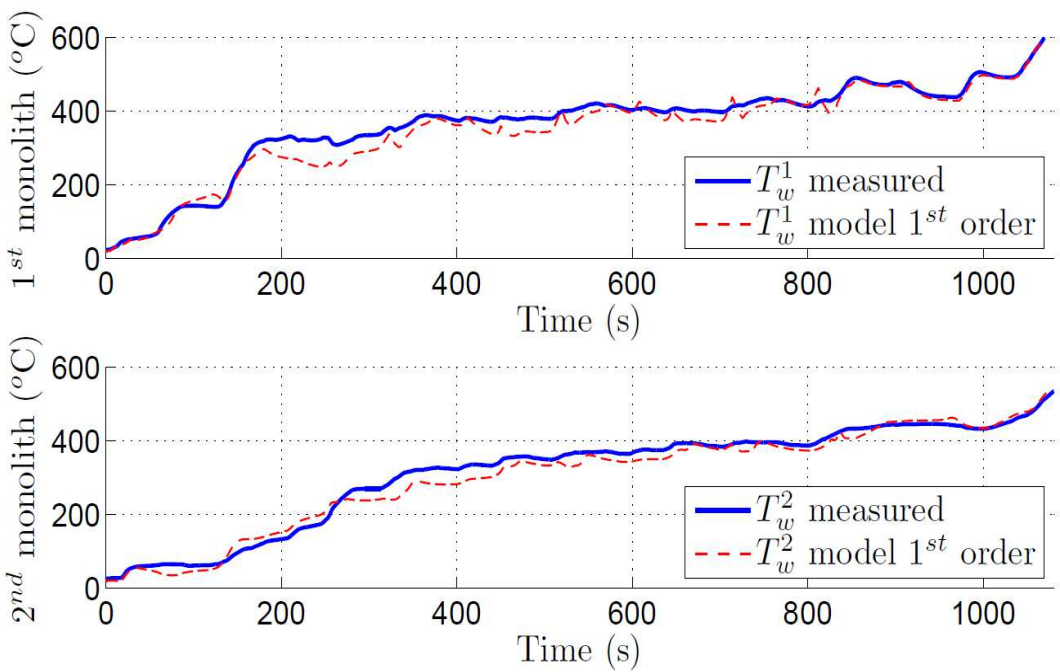

(a) Wall temperature at two different locations

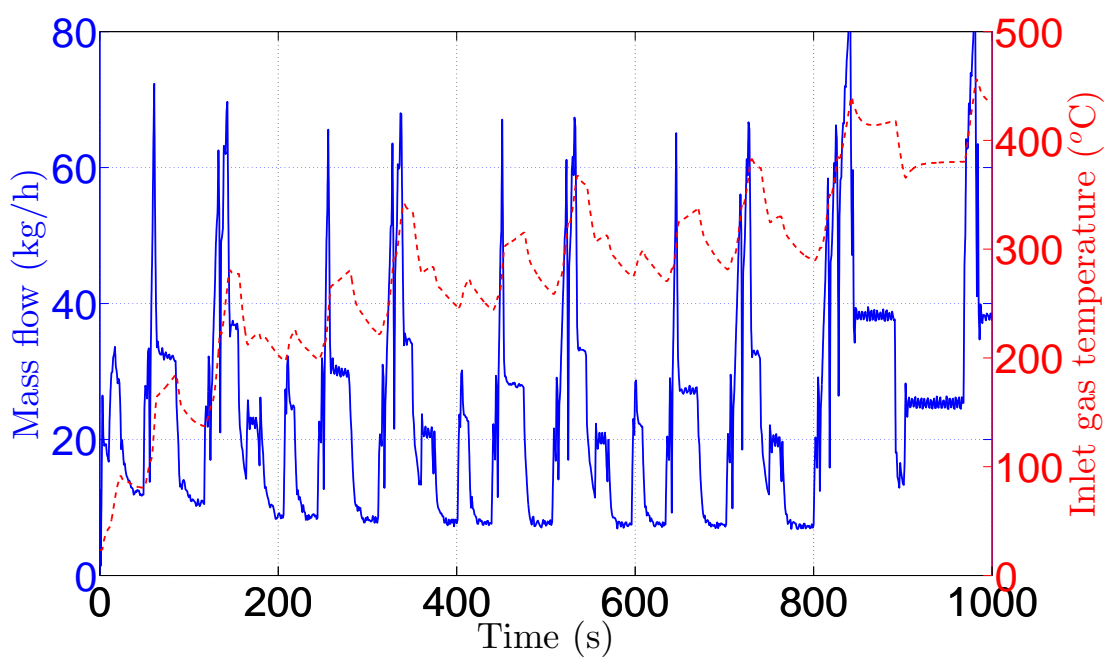

(b) Exhaust gas flow and temperature.

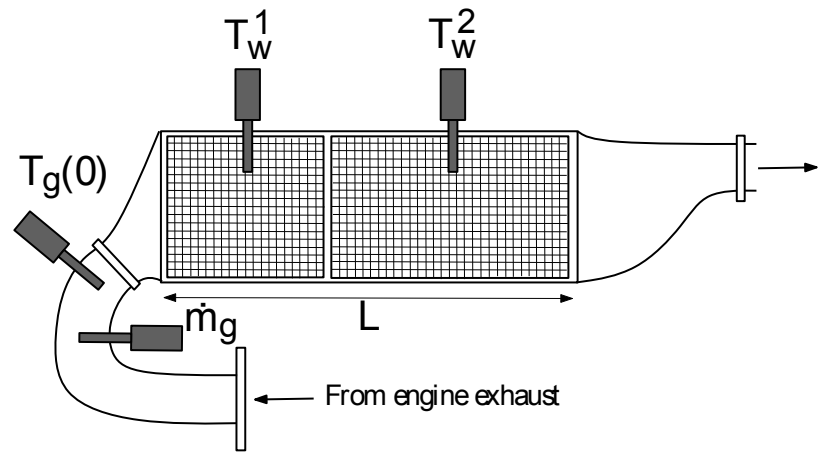

(c) Available sensors with their location in test-bench facilities

Fig. 4 Comparison between experimental data and simulation results obtained with the reduced first-order input-delay model (6)-(11). Wall temperature at two different locations pictured in 4(c) and for the inputs represented in 4(b) are reported in 4(a). 
actuator, the EGR valve, located upstream of the compressor and the inlet manifold). This leads to a large transport delay (up to several seconds depending on the engine specifications) which can be modeled by an integral relation of type (1).

The main difficulty when using this representation arises from thermal exchanges and changes in gas velocity occurring in the intake line, which are not directly caught by (1). We aim here at illustrating how this complexity can be handled by presenting a relatively fair practical delay calculation methodology to account for these phenomena. This procedure is compliant with real-time constraints and has been experimentally validated on a test bench.

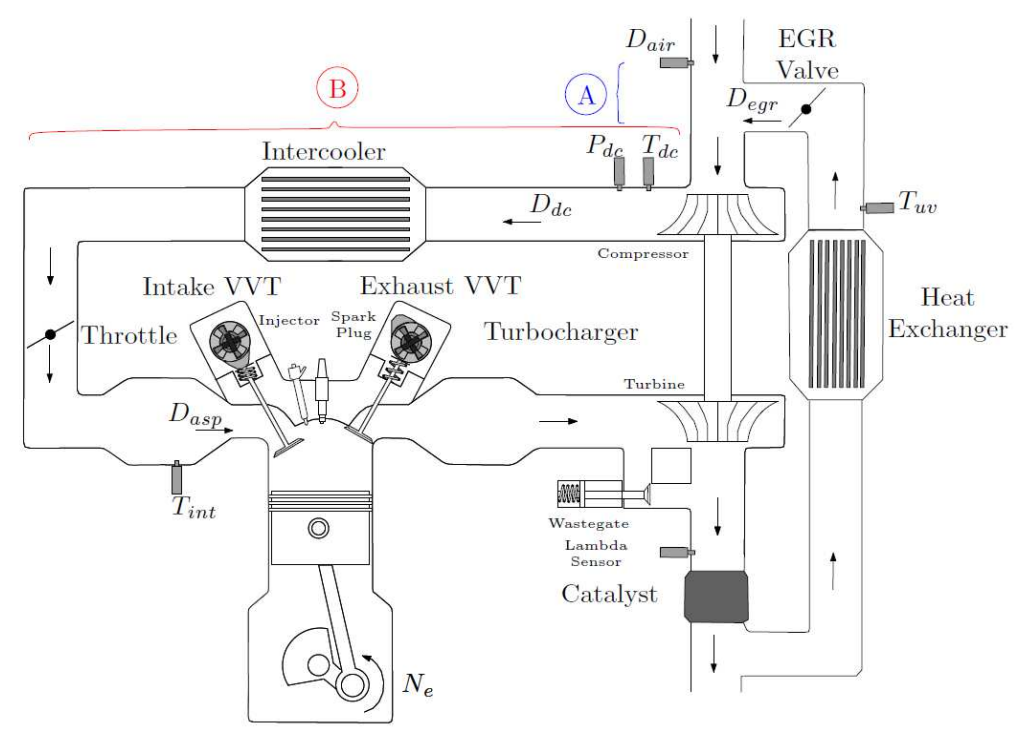

Fig. 5 Scheme of a turbocharged SI engine equipped with direct injection, VVT and a low-pressure EGR loop.

\subsection{Modeling}

Formally, the in-cylinder burned gas fraction $x_{c y l}$ is defined as the ratio between the in-cylinder burned gas mass originated from the EGR loop $m_{b g}$ and the total mass of gas in the cylinder volume $m_{a s p}=m_{a i r}+m_{b g}$, i.e.

$$
x_{c y l}=\frac{m_{b g}}{m_{a i r}+m_{b g}}
$$

From now on, this variable is considered equal to $x$ the intake burned gas fraction ${ }^{6}$.

\footnotetext{
${ }^{6}$ Actually, this relation depends mainly on the Variable Valve Actuator control strategy. We neglect this influence here for sake of clarity.
} 
EGR burned gas

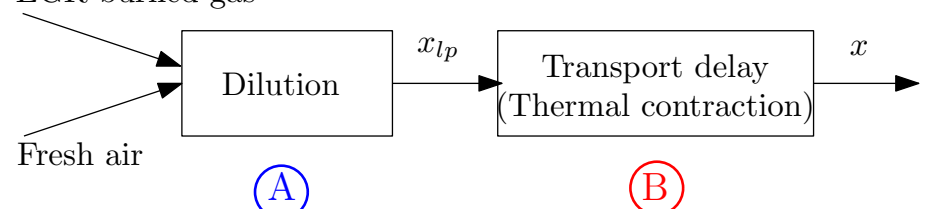

Fig. 6 Scheme of the intake burned gas fraction dynamics.

Defining $x_{l p}$ as the burned gas rate upstream of the compressor, the EGR dynamics can be expressed as

$$
\begin{aligned}
\dot{x}_{l p} & =\alpha\left[-\left(D_{\text {egr }}(t)+D_{a i r}(t)\right) x_{l p}(t)+D_{\text {egr }}(t)\right] \\
x(t) & =x_{l p}(t-D(t))
\end{aligned}
$$

where $\alpha$ is a positive constant, $D_{\text {air }}$ is the inlet air mass flow rate, $D_{\text {egr }}$ is the recirculated exhaust gas mass flow rate and $D(t)$, the delay between this ratio and the intake composition, satisfies

$$
\int_{t-\tau(t)}^{t} v_{g a s}(s) d s=L_{P}
$$

where $L_{P}$ is the pipe length from the compressor down to the intake manifold and $v_{\text {gas }}$ stands for the gas speed.

Equation (12) is a balance equation on the volume downstream of the EGR valve, using the fact that the EGR circuit is totally filled with burned gas ${ }^{7}$. According to engine setups, the thermodynamics constant $\alpha$ appearing in (12) is either measured or known. Following the proposed model, which is pictured in Fig. 6, the intake burned gas fraction is the result of a first order dynamics coupled with a transport delay. This transport delay is of the form (1) with $\varphi(s, u(s))=\frac{v_{g a s}}{L_{P}}$. Because the velocity of the gas is indirectly related to the amount of reintroduced burned gas, which is the control variable, this delay is input-dependent, as will reveal in the following.

For sake of clarity, the approach used to model the mass flow rate quantities $\left(D_{e g r}, \ldots\right)$ used through (12) to provide an open-loop estimator of the variable $x_{l p}$ is not detailed here. The interested reader can refer to [6] for further details. In the following, we assume that such an estimator $\hat{x}_{l p}$ and the different mass flow rates are available.

To provide an implementable open-loop estimate of $x$ based on the model (12)(14), a practical calculation methodology of the delay $D$, using only real-time mea-

\footnotetext{
${ }^{7}$ For SI engines, the FAR is regulated to its stoichiometric value (see [19]), which results into an exhaust burned gas fraction close to unity. Note that this assumption disrupts the potential link that could exist between the intake manifold composition and the exhaust line one, which could create an implicit loop in our approach.
} 
surements, remains to be developed. Indeed, the velocity of the gas is not a measured quantity. Further, the thermodynamical transformations the gas is submitted to in the intake line impact this velocity. These variations need to be taken into account. We now address this point.

\subsection{Transport delay description}

Using the ideal gas law (as is classically done for engine gas flows, e.g. in [19]), one can relate the gas speed to current thermodynamical conditions and mass flow rates, which are measured/modeled. Namely,

$$
\forall t \geq 0, \quad v_{\text {gas }}(t)=\frac{1}{S(t)} \frac{r T(t)}{P(t)}\left[D_{\text {air }}(t)+D_{\text {egr }}(t)\right]
$$

where $S$ is the current pipe area, $T, P$ are the current temperature and pressure values, $r$ is, as previously, the (common) ideal gas constant of both fresh air and burned gas.

A thermal contraction of the gas occurs inside the intake cooler. This results in spatial changes of the gas velocity $v_{\text {gas }}$, pictured in Fig.5. To model this, we split the intake line into three main sections with three respective and cumulative transport delays $D_{1}, D_{2}$ and $D_{3}$ such that $D=D_{1}+D_{2}+D_{3}$.

- downstream of the compressor to the intercooler: in this part, the current pressure and the temperature are measured and, respectively, are equal to $P_{d c}$ and $T_{d c}$. Then, one can write

$$
\int_{t-D_{1}(t)}^{t} \frac{r T_{d c}}{P_{d c}}\left[D_{a i r}(s)+D_{e g r}(s)\right] d s=V_{1}
$$

with $V_{1}$ the corresponding volume.

- inside the intercooler: considering boundary conditions, the pressure inside the intercooler can reasonably be assumed as constant and equal to the input one $P_{d c}$. Further, we assume that the spatial profile of the inside temperature is affine with respect to the spatial variable, with measured boundary conditions $T_{d c}$ and $T_{\text {int }}{ }^{8}$. Under this assumption, equation (14) can be reformulated on this section as

$$
\begin{gathered}
\int_{t-D_{2}(t)-D_{1}(t)}^{t-D_{1}(t)} \frac{r}{P_{d c}}\left[D_{a i r}(s)+D_{e g r}(s)\right] d s=S_{2} \int_{0}^{L_{2}} \frac{d x}{T(x)} \\
=\frac{V_{2}}{T_{i n t}-T_{d c}} \ln \left(\frac{T_{\text {int }}}{T_{d c}}\right)
\end{gathered}
$$

where $L_{2}, S_{2}$ and $V_{2}$ are the corresponding length, area and volume.

${ }_{8 \text { i.e. } T(x)=\frac{T_{i n t}-T_{d c}}{L_{2}} x+T_{d c}}$ 
- downstream of the intercooler to the intake manifold: in this section, the temperature can be approximated by the intake manifold temperature $T_{\text {int }}$, which yields

$$
\int_{t-D_{3}(t)-D_{2}(t)-D_{1}(t)}^{t-D_{2}(t)-D_{1}(t)} \frac{r T_{i n t}}{P_{d c}}\left[D_{a i r}(s)+D_{e g r}(s)\right] d s=V_{3}
$$

with $V_{3}$ the corresponding volume.

Knowing intermediate volumes $V_{1}, V_{2}$ and $V_{3}$, one can calculate the delay in a very straightforward manner, solving, one after the other, (15), (16) and finally (17). The transport delay is then simply deduced as $D(t)=D_{1}(t)+D_{2}(t)+D_{3}(t)$. The numerical solving of these equations is addressed in Section 2.2 and involves realtime compliant operations. Real-time measurements of temperatures and pressures serve to determine the value of the delay. These information are commonly available using embedded sensors. Values for physical volumes $\left(V_{1}, V_{2}\right.$ and $\left.V_{3}\right)$ can be used to calibrate the model.

\subsubsection{Experimental use of the proposed model}

The modeling (12)-(13) can be used to estimate online the intake burned gas rate. In details, through the delay calculation methodology proposed in the previous section, one can build a "software" sensor, embedded into a real-time control target and employed at test-bench. Experiments validating the delay modeling and, in particular, the integral delay model have been carried out. They are presented in [7].

\section{Control stakes and open problems}

As mentioned in [37], while a few works have investigated open-loop design for input-dependent delay systems (see for example [13-15] which investigates a delay model of type type (1)), closed-loop control for input-dependent delay systems is still an open problem. The main difficulty arises from the fact that the delay is not only varying but also depends on the control variable in a way which should be taken into account while designing the feedback law.

In a nutshell, for time-varying delays, available techniques either employ a frequency technique to study the sensitivity of the infinite number of roots to delay variations [31] or solve Linear Matrix Inequalities (LMI) to ensure stabilization [18]. Both techniques determine admissible feedback gain depending on a upper-bound of the delay variations. Nevertheless, when the delay depends on the input, things get very involved. Indeed, delay variations are then related to feedback gain and designing a stabilizing feedback law using these techniques becomes an implicit issue. 
In this section, we present some preliminary but encouraging directions of work for input-dependent delay of type (1).

\subsection{Including the delay in the system state}

For sake of simplicity, consider the non-linear dynamics

$$
\dot{x}(t)=f(x(t), x(t-D(t)), u(t), u(t-D(t)))
$$

in which $D(t)$ is defined through (1). Then, taking a time-derivative of (1), and defining an extended state $z=\left[\begin{array}{ll}x & z_{n+1} \triangleq D\end{array}\right]^{T}$, (18) rewrites

$$
\dot{z}=\left(\begin{array}{c}
f\left(x(t), x\left(t-z_{n+1}\right), u(t), u\left(t-z_{n+1}\right)\right) \\
1-\frac{\varphi(t, u(t))}{\varphi\left(t-z_{n+1}, u\left(t-z_{n+1}\right)\right)}
\end{array}\right)
$$

Then, this transformation allows to transform an input-varying delay into a statedependent delay, which is less complex to analyse. However, this configuration problem is not directly tractable using currently available tools for state-dependent input-delays because the extra delay differential equation brings controllability questions. One possibility could be to blend this formulation with the successive interval approach employed in [15] to calculate admissible trajectories.

\subsection{Prediction-based controller for input-delay}

Predictor-based control strategies $[1,29,39]$ have been proposed for time-varying delay systems (see [32] or, more recently, in [24]). The idea of this technique is to compensate the input delay by calculating a state prediction over a time window of which length matches the value of the future delay. Namely, consider the following linear plant

$$
\begin{aligned}
\dot{x}(t) & =A x(t)+B u(r(t)) \\
r(t) & =t-D(t)
\end{aligned}
$$

with $x \in \mathbb{R}^{n}$ and $u \in \mathbb{R}^{m}$. Then, compensation of the delay is achieved by using the control law

$$
u(t)=K x\left(r^{-1}(t)\right)
$$

in which the feedback gain $K$ is stabilizing. In other words, one needs to predict the future variations of the delay to calculate the time horizon $r^{-1}(t)$. For example, this is the approach followed in [41] for a time-varying delay, the variations of which are provided by a given known model. It has also been extended in [2,3] to nonlinear 
dynamics and state-dependent delay, where variations are characterized by a careful prediction of the system state.

Nevertheless, determination of such an horizon may not be practically achievable when the delay depends on the input, because of the reciprocal interactions between the control (current and past) values and the delay. The authors recently proposed in [5] to use the current delay value as prediction horizon. To analyse the closedloop stability, a two-step methodology was proposed to disrupt the loop between input and delay. For an input delay satisfying

$$
\int_{t-D(t)}^{t} u(s) d s=1
$$

it was shown that robust prediction-based stabilization is achieved provided that the feedback magnitude gain is sufficiently small, according to the initial condition scale.

This methodology consists in the following steps:

- In a first move, the input-dependent delay is considered as an element of the more general class of time-varying delay. It enables then to use a robust compensation result which has been obtained for linear systems with time-varying input delay, using the backstepping tools proposed in [23] for the analysis of input-delay systems stability. Technically, this result guarantees stabilization provided the delay variations are sufficiently small.

- In a second step, a more practical sufficient condition for stabilization is obtained by relating the delay variations to the control tracking error, which is analysed using the asymptotic convergence of delay differential equations (DDE) (Halanay-type inequalities [16]). The final sufficient conditions characterizes the admissible feedback gain magnitude according to the initial condition scale.

Extension of this technique to the general delay model (1) is an important challenge that remains to be addressed. Further, stabilization of systems with state delays of type (1) is also a major question to be explored.

\section{Conclusion}

This chapter focused on a particular integral-type delay model, which is representative of a large class of transport processes and yet still understudied in Automatic Control. This last point was highlighted through the modeling of two subsystems from the field of automotive engine control, the dynamics of which involve a transport delay and can be modeled accordingly. Experimental relevance of the proposed model has been shown. Yet, various challenges remain while aiming at controlling such systems. In particular, the input-dependence of the delay integral is often nonnegligible and should be taken into account. This point is still an open problem. 


\section{References}

1. Z. Artstein. Linear systems with delayed controls: a reduction. IEEE Transactions on Automatic Control, 27(4):869-879, 1982.

2. N. Bekiaris-Liberis and M. Krstic. Compensation of state-dependent input delay for nonlinear systems. In Conference on Decision and Control, 2011.

3. N. Bekiaris-Liberis and M. Krstic. Compensation of time-varying input and state delays for nonlinear systems. Journal of Dynamic Systems, Measurement and Control, 134, 2012. Paper 011009.

4. N. Bekiaris-Liberis and M. Krstic. Nonlinear control under delays that depend on delayed states. European Journal on Control, Special Issue for the ECC13, 19:389-398, 2013.

5. D. Bresch-Pietri, J. Chauvin, and N. Petit. Prediction-based Stabilization of Linear Systems Subject to Input-Dependent Input Delay of Integral-Type. In IEEE Transactions on Automatic Control, 2014, Vol. 59, pages 2385-2399.

6. D. Bresch-Pietri, T. Leroy, J. Chauvin, and N. Petit. Prediction-based trajectory tracking of external gas recirculation for turbocharged SI engines. In Proc. of the American Control Conference, 2012.

7. D. Bresch-Pietri, T. Leroy, J. Chauvin, and N Petit. Practical delay modeling of externally recirculated burned gas fraction for spark-ignited engines. In 11th Workshop on Time-Delay Systems, 2013.

8. D. Bresch-Pietri, T. Leroy, and N Petit. Control-oriented time-varying input-delayed temperature model for SI engine exhaust catalyst. In American Control Conference, pages 1583-1590, 2013.

9. D. Bresch-Pietri, T. Leroy, and N. Petit. Estimation of the distributed temperature of a SI engine catalyst for light-off strategy. In European Control Conference, 2013.

10. M. Chèbre, Y. Creff, and N. Petit. Feedback control and optimization for the production of commercial fuels by blending. Journal of Process Control, 20(4):441-451, 2010.

11. P. V. Danckwerts. Continuous flow systems: distribution of residence times. Chemical Engineering Science, 2(1):1-13, 1953.

12. C. Depcik and D. Assanis. One-dimensional automotive catalyst modeling. Progress in energy and combustion science, 31(4):308-369, 2005.

13. J.-Y. Dieulot, N. Petit, P. Rouchon, and G. Delaplace. An arrangement of ideal zones with shifting boundaries as a way to model mixing processes in unsteady stirring conditions in agitated vessels. Chemical Engineering Science, 60:5544-5554, 2005.

14. J.-Y. Dieulot, N. Petit, P. Rouchon, and G. Delaplace. A torus model containing a sliding wellmixed zone as a way to represent mixing process at unsteady stirring conditions in agitated vessels. Chemical Engineering Communications, 192:805-826, 2005.

15. J.-Y. Dieulot and J.-P. Richard. Tracking control of a nonlinear system with input-dependent delay. Proc. of the 40th Conference on Decision and Control, 4:4027-4031, 2001.

16. A. Halanay. Differential Equations: Stability, Oscillations, Time Lags, volume 23. Academic Press, 1966.

17. J. Harmand and D. Dochain. The optimal design of two interconnected (bio) chemical reactors revisited. Computers \& chemical engineering, 30(1):70-82, 2005.

18. Y. He, Q. G. Wang, C. Lin, and M. Wu. Delay-range-dependent stability for systems with time-varying delay. Automatica, 43(2):371-376, 2007.

19. J. B. Heywood. Internal Combustion Engine Fundamentals. McGraw-Hill New York, 1988.

20. B. Hoepke, S. Jannsen, E. Kasseris, and W. K. Cheng. EGR effects on boosted SI engine operation and knock integral correlation. SAE International Journal of Engines, 5(2):547$559,2012$.

21. M. Jankovic and I. Kolmanovsky. Developments in control of time-delay systems for automotive powertrain applications. In Delay Differential Equations, recent advances and new directions, Balachandran, B. and Kalmar-Nagy, T. and Gilsinn D. E., pages 55-92. Springer Science, 2009.

22. U. Kiencke and L. Nielsen. Automotive Control Systems. Springer-Verlag, Berlin, 2000. 
23. M. Krstic. Boundary Control of PDEs: a Course on Backstepping Designs. Society for Industrial and Applied Mathematics Philadelphia, PA, USA, 2008.

24. M. Krstic. Delay Compensation for Nonlinear, Adaptive, and PDE Systems. Birkhauser, 2009.

25. D. Kum, H. Peng, and N. K. Bucknor. Optimal energy and catalyst temperature management of plug-in hybrid electric vehicles for minimum fuel consumption and tail-pipe emissions. IEEE Transactions on Control Systems Technology, 21(99):14-26, 1999.

26. P. M. Laing, M. D. Shane, S. Son, A. A. Adamczyk, and P. Li. A simplified approach to modeling exhaust system emissions: SIMTWC. SAE paper, -:01-3476, 1999.

27. O. Lepreux. Model-based temperature control of a Diesel oxidation catalyst. PhD thesis, MINES Paristech, 2010.

28. O. Lepreux., Y. Creff, and N. Petit Model-based temperature control of a Diesel oxidation catalyst. Journal of Process Control, 22:41-50, 2012.

29. A. Manitius and A. Olbrot. Finite spectrum assignment problem for systems with delays. IEEE Transactions on Automatic Control, 24(4):541-552, 1979.

30. MATHWORKS. http://www.mathworks.com/.

31. W. Michiels, V. Van Assche, and S. I. Niculescu. Stabilization of time-delay systems with a controlled time-varying delay and applications. IEEE Transactions on Automatic Control, 50(4):493-504, 2005.

32. M. T. Nihtila. Finite pole assignment for systems with time-varying input delays. In Proc. of the 30th IEEE Conference on Decision and Control, pages 927-928, 1991.

33. A. Onorati, G. D'Errico, and G. Ferrari. 1D fluid dynamic modeling of unsteady reacting flows in the exhaust system with catalytic converter for SI engines. SAE Transactions, 109(200001-0210):89-103, 2000.

34. R. H. Perry, D. W. Green, and J. O. Maloney. Perry's Chemical Engineers' Handbook, volume 7. McGraw-Hill New York, 1984.

35. N. Petit, Y. Creff, and P. Rouchon. Motion planning for two classes of nonlinear systems with delays depending on the control. In Proceedings of the 37th IEEE Conference on Decision and Control, pages 1007-1011, 1998.

36. S. Potteau, P. Lutz, S. Leroux, S. Moroz, et al. Cooled EGR for a turbo SI engine to reduce knocking and fuel consumption. In SAE Technical Paper, pages 01-3978, 2007.

37. J.-P. Richard. Time-delay systems: an overview of some recent advances and open problems. Automatica, 39(10):1667-1694, 2003.

38. M. Sbarciog, R. De Keyser, S. Cristea, and C. De Prada. Nonlinear Predictive Control of processes with variable time delay. A temperature control case study. In Proc. of the IEEE International Conference on Control Applications, pages 1001-1006, 2008.

39. O. J. M. Smith. A controller to overcome dead time. ISA Journal, 6(2):28-33, 1959.

40. J. Vardi and W. F. Biller. Thermal behavior of exhaust gas catalytic convertor. Industrial \& Engineering Chemistry Process Design and Development, 7(1):83-90, 1968.

41. E. Witrant. Stabilisation des systèmes commandés par réseaux. PhD thesis, Laboratoire d'Automatique de Grenoble, 2005.

42. M. Wu, C. Wang, W. Cao, X. Lai, and X. Chen. Design and application of generalized predictive control strategy with closed-loop identification for burn-through point in sintering process. Control Engineering Practice, 20:1065-1074, 2012.

43. L. C. Young and B. A. Finlayson. Mathematical models of the monolith catalytic converter. AIChE Journal, 22(2):343-353, 1976.

44. D. Yue and Q. L. Han. Delayed feedback control of uncertain systems with time-varying input delay. Automatica, 41(2):233-240, 2005.

45. K. Zenger and A. J. Niemi. Modelling and control of a class of time-varying continuous flow processes. Journal of Process Control, 19(9):1511-1518, 2009.

46. K. Zenger and R. Ylinen. Simulation of variable delays in material transport models. Mathematics and computers in simulation, 37:52-72, 1994. 